\title{
Rapid 3D imaging of the airway by MRI in patients with congenital heart disease: comparison of delayed volume interpolated breath hold examination (VIBE) technique to the turbo spin echo (TSE)
}

\author{
Benjamin Goot ${ }^{*}$ Sonali Patel, Brian Fonseca
}

From 17th Annual SCMR Scientific Sessions

New Orleans, LA, USA. 16-19 January 2014

\section{Background}

When imaging the airway by MRI, the traditional technique turbo spin echo (TSE) results in high quality $2 \mathrm{D}$ images of the airway. However, planning and acquisition times are often lengthy. An alternative technique for airway image acquisition, delayed volume interpolated breath hold examination or VIBE, is a 3D gradient echo technique that produces high spatial resolution imaging of the airway in one breath hold. The aim of this study was to compare airway measurements obtained by both TSE and delayed VIBE sequences in order to demonstrate that the quicker delayed VIBE produces a comparable evaluation of the airway to the standard technique.

\section{Methods}

Patients with congenital heart disease, who underwent a cardiac MRI that included a delayed VIBE sequence from $5 / 2008$ to the $9 / 2013$, were included. Studies were performed on a 1.5-T Siemens Avanto (Siemens Medical Solutions, Erlagen, Germany). The TSE imaging was performed in the axial, sagittal and coronal planes per institutional protocol. The delayed VIBE (TR/TE $3.7 / 1.2 \mathrm{~ms}$, flip angle 150 , voxel $1.5-1.8 \times 0.8-1 \times 1.0-1.2 \mathrm{~mm}$ ) was acquired in the sagittal plane 5 minutes after gadolinium contrast administration. Two observers reviewed the studies (BG, BF) and airway measurements were made on both the delayed VIBE and TSE images in a blinded fashion to the other observer and other technique values.

\footnotetext{
Pediatrics-Division of Cardiology, University of Colorado-Children's Hospital
} Colorado, Aurora, Colorado, USA

Intraclass correlations were calculated for agreement between both techniques, as well as between the two observers.

\section{Results}

29 studies met inclusion criteria for analysis with a mean age of 8.8 years ( 2 months to 63 years) and mean weight of $30.2 \mathrm{~kg}$ (3.5-110). Of those 29 studies, 16 had both TSE and delayed VIBE sequences. The remaining 13 studies had no TSE imaging but the delayed VIBE measurements were included in the analysis for inter-observer variability. All delayed VIBE and TSE sequences were found to be of diagnostic quality. Mean acquisition time was significantly shorter for the delayed VIBE at $13.1 \mathrm{sec}-$ onds (7.6-24.9) than TSE at 949.9 seconds (361-2318). Overall there was very good agreement between the delayed VIBE and TSE measurements (intraclass correlacoefficient (ICC) 0.94-0.78) with the exception of the distal right bronchus (ICC 0.67) The inter-observer agreement was also excellent for both TSE (ICC 0.94-0.78) and VIBE (ICC 0.96-0.89) (see Table 1).

\section{Conclusions}

Delayed VIBE is a rapid and robust alternative to TSE imaging for assessment of the airway by MRI across a wide spectrum of patient age and size.

\section{Funding}

This study was not funded and the author(s) declare no potential conflicts of interest with respect to the research, authorship, and/or publication of this article. 
Table 1

\begin{tabular}{|c|c|c|c|c|}
\hline \multirow[b]{2}{*}{ Site } & \multicolumn{2}{|c|}{ Inter-technique ICC by Observer } & \multicolumn{2}{|c|}{ Inter-observer ICC by Technique } \\
\hline & BG & $\mathrm{BF}$ & TSE & VIBE \\
\hline Proximal Trachea Major Axis & 0.871 & 0.921 & 0.897 & 0.924 \\
\hline Proximal Trachea Minor Axis & 0.899 & 0.919 & 0.917 & 0.921 \\
\hline Proximal Trachea Area & 0.915 & 0.782 & 0.900 & 0.851 \\
\hline Distal Trachea Major Axis & 0.782 & 0.900 & 0.938 & 0.903 \\
\hline Distal Trachea Minor Axis & 0.810 & 0.878 & 0.886 & 0.932 \\
\hline Distal Trachea Area & 0.783 & 0.882 & 0.914 & 0.948 \\
\hline Proximal Right Bronchus & 0.936 & 0.801 & 0.956 & 0.955 \\
\hline Distal Right Bronchus & 0.917 & 0.666 & 0.803 & 0.928 \\
\hline Proximal Left Bronchus & 0.792 & 0.855 & 0.834 & 0.912 \\
\hline Distal Left Bronchus & 0.890 & 0.795 & 0.779 & 0.894 \\
\hline
\end{tabular}

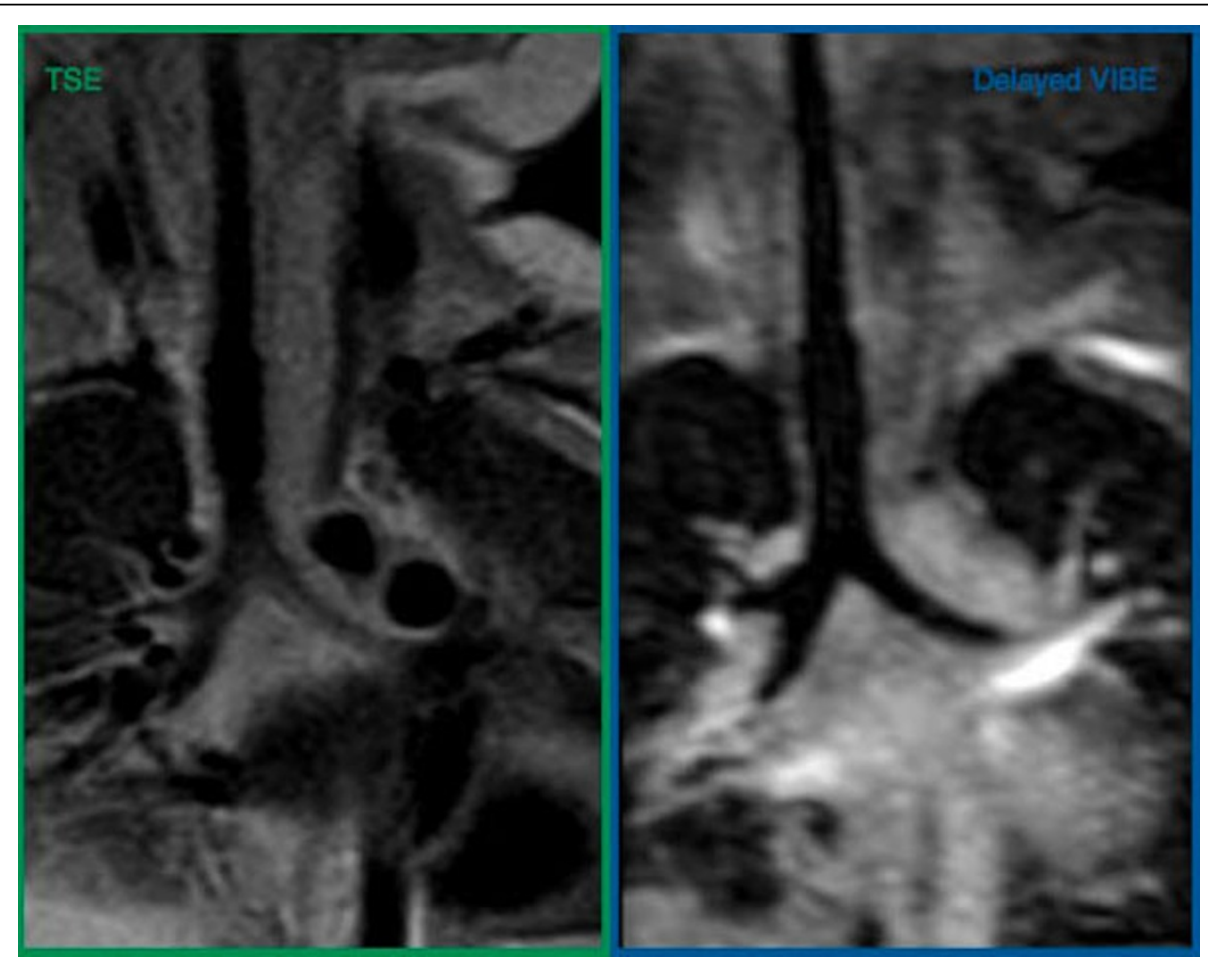

Figure 1 Coronal images from a 1 year 11 month old child $(7 \mathrm{~kg})$ demonstrating the image quality obtained from both TSE and VIBE.

Published: 16 January 2014

doi:10.1186/1532-429X-16-S1-P117

Cite this article as: Goot et al:: Rapid 3D imaging of the airway by MRI in patients with congenital heart disease: comparison of delayed volume interpolated breath hold examination (VIBE) technique to the turbo spin echo (TSE). Journal of Cardiovascular Magnetic Resonance 2014 16(Suppl 1):P117

\section{Submit your next manuscript to BioMed Central} and take full advantage of:

- Convenient online submission

- Thorough peer review

- No space constraints or color figure charges

- Immediate publication on acceptance

- Inclusion in PubMed, CAS, Scopus and Google Scholar

- Research which is freely available for redistribution 